\title{
South Asian immigrant women's experiences of being respected within cancer treatment settings
}

by Savitri Singh-Carlson, Anne Neufeld and Joanne Olson

\begin{abstract}
The purpose of this focused ethnographic inquiry was to examine South Asian immigrant women's experiences and perceptions of respect within health professional-client relationships in the context of a Canadian outpatient treatment clinic. Characteristics of respect described by 11 women interviewed were the meaning of respect, health professional's way of being, their way of attending to the person, and their way of talking. Language, cultural values and beliefs, along with underlying societal, individual and institutional factors that coexist with health professionals' ability to create respect were some of the dimensions that influenced how immigrant women experienced respect. Health professionals' capacity to acknowledge South Asian immigrant women as individuals helped to formulate/construct respect for their individual identities. The need to be respected for 'my social identity' as an immigrant woman with cancer was woven throughout women's stories, illustrated by their personal experiences and perspectives.
\end{abstract}

A demographic shift in Canada within the past two decades has resulted in a substantial increase in immigration from non-European countries (Statistics Canada, n.d.). Immigrant women, women refugees, and women of diverse ethnic backgrounds form an increasingly large percentage of the Canadian population (Statistics Canada, n.d.). The resulting population heterogeneity presents challenges to the provision of health care for people living with chronic, as well as life-threatening illnesses such as cancer (Kirkham, 2003).

Based on current incidence rates, 39\% of women will develop cancer at some point during their lifetime (Canadian Cancer Society, 2008). On average, 1,319 Canadian women were newly diagnosed and 608 women died of cancer every week in 2004 (Canadian Cancer Society, 2008). A comparison of profiles of Indian women residing in India and Canada reported high incidence rates for breast cancer for both groups of women, with higher incidence rates for cervical cancer for British Columbia Indo-Canadian women in comparison to the B.C. general population (Hislop et. al., 2007).

\section{Background}

Despite extensive discussions in the literature about respect as an ethical and moral concept (Benner, 2002; Milton, 2008; Tarlier, 2004) and a fundamental component of providing care in the practice setting (Barbosa \& Silva, 2007; Erlen, 1998), few studies have critically examined how respect is conveyed within the nurse-client domain of nursing (Browne, 1995, 1997; Kelly, 1990). Even fewer studies have examined the experience of being respected from the client's perspective within health professional-client relationships in health care settings, especially for immigrant women. Understanding the

\section{About the authors \\ Savitri Singh-Carlson, BSN, PhD, Assistant Professor, California State University Long Beach, 1250 Bellflower Boulevard, Long Beach, CA USA 90803. Phone: 562230 1016; Fax: 562985 2382; Email: scarlson@csulb.edu}

Anne Neufeld, RN, PhD, Professor, Faculty of Nursing, University of Alberta, Edmonton, $A B$.

Joanne Olson, RN, PhD, Professor \& Associate Dean, Undergraduate Programs, Faculty of Nursing, University of Alberta, Edmonton, $A B$. range of social, historical, individual, and political factors that affect health care interactions is vital if health care providers are to provide respectful care, which will prevent discrimination and dehumanization that marginalizes multicultural patients when they enter the mainstream health care system (Browne, 1995, 1997).

Respectful relationships reduce differences between individuals and shape the way in which people engage with each other (Gallagher, 2007). A recent study with Canadian South Asian immigrant women indicated that the initial breast cancer diagnosis experience is complex, involving individual cognitive processes and negotiations with others, including health care professionals (Bottorff et al., 2007). Although most native-born Canadian women will confront various challenges with cancer, these challenges are usually heightened for immigrant women who might be struggling with migration and settlement issues, including the challenges of maintaining lifelong beliefs and practices while learning new ways to establish a stable life in the new country (Gupta, Kumar, \& Stewart, 2002; Hilton et al., 2001). For example, immigrant women must deal with the cancer diagnosis as well as other resettlement issues, such as leaving their extended family behind in their country of origin, or caring for other family members, and adjusting to their life in Canada (Hilton et al., 2001; Clegg, 2003; Singh, 2002).

Health-related disciplines, including nursing, medicine, the counselling professions, and social work all identify respect as a central ethical principle that guides clinical practice (Beauchamp \& Childress, 1994; Morris, 1997). Although the foundation of nursing care and nursing principles and values such as autonomy, dignity and integrity have been built on the concept of respect (Klutgen, 1995; Widäng, Fridlund, \& Martensson, 2007), these concepts have been used in the literature synonymously with respect to explicate the ethical dimension of respect (Peters, 1961; Rokeach, 1979). The aim in this qualitative study was to examine respect, a positive component of health professional-client relationships which plays a major role in promoting health and well-being, along with the individual's attaining and retaining an optimum level of health, regardless of ethnicity or gender (Acton et al., 1997).

\section{Purpose}

The purpose of this inquiry was to examine South Asian immigrant women's experiences and perceptions of respect within health professional-client relationships while undergoing treatment for cancer. Women who came to a health care agency outpatient followup clinic for cancer-related treatments participated in the study.

\section{Method}

A focused ethnographic approach (Roper \& Shapiro, 2000) informed by Dorothy E. Smith's (1987) feminist standpoint perspective addressed South Asian immigrant women's meanings of respect in relationships with health professionals in the social setting of a cancer clinic. Ethnography, a naturalistic approach was appropriate for this study because of its broad use in health care research as a means of understanding individuals' experiences of illness, their cultural health beliefs and practices, and patterns of behaviour within the social context of their lives (Morse \& Field, 1995). To provide thick descriptions or rich narratives of participants' experiences, which is the main goal of this qualitative approach, intense field work included participant observation, face-to-face in-depth interviews, detailed field notes and journaling as the principal sources of data. This helped to establish scientific rigour by demonstrating trustworthiness, auditability, and credibility in this qualitative inquiry. Reflexivity during data collection and analysis helped to reduce researcher bias within the findings. 
The study was approved by the joint health research ethics review boards of the university and the cancer agency. Recruitment from two local cancer clinics was done by charge nurses and radiation therapists by providing women with an information package, and allowing interested women to contact the researcher directly. Inclusion criteria were South Asian immigrant women 18 years of age or older who were diagnosed with cancer and were, at present, receiving treatments or coming for follow-up clinical visits. They were not palliative. For the purposes of this study, South Asian immigrant women were defined as those of Indian descent who had immigrated to Canada from Fiji Islands, India, Pakistan, or Sri Lanka.

\section{Data collection and analysis}

Health professionals of each discipline who cared for cancer clients were shadowed over a period of two weeks for three to four hours each time before initiating the interviews to observe and identify daily morning and afternoon routine activities of the settings. The intent was not to evaluate health professionals or disciplines in their relationships with clients, but to use participant observations as an entry point to understanding relationships in the wider context of health professionals' everyday work. The observations were not restricted to observing cancer care for only South Asian immigrant women, but for all the clients visiting the clinics. Further participant observations at various points were conducted in order to clarify, confirm, and validate contextual data.

Face-to-face interviews conducted by the first author lasted 30 minutes to an hour in women's homes or another location of their choice. Interview questions were piloted with two South Asian immigrant women who were ineligible for the study (did not have cancer), but were dealing with other chronic illnesses. Revisions to the semistructured interview guiding questions enabled women to tell their stories freely. Although our primary goal was to understand participants' positive experiences and perceptions of respect, we included questions regarding situations of disrespect or a lack of respect because they may coexist within health care relationships, as the converse of respectful situations. Four women who described a range of perspectives on respect were purposively approached and interviewed a second time in order to elaborate on, validate and confirm their accounts and clarify variations in the data. Questions for the second interviews were generated from review and analysis of data from women's first interviews. This strategy helped to gain further insight that was useful in exploring and clarifying themes and aspects of the participant's differing and similar experiences of respect. Browne $(1995,1997)$ found that interviewing informants a second time provided confirmation and validation of the data, as well as adding clarity to an understanding of the meanings of respect among women. Some family members, for example, husbands or daughters, facilitated the interview process because they were the primary caregivers. Written consent forms in English and translated versions in Punjabi or Hindi were presented to participants prior to the interview.

A constant comparative analysis (Morse \& Field, 1995) of the incoming data was done to identify recurring, converging and contradictory patterns of women's experiences, preliminary themes and categories, and to select illustrations from the data. Interviews were audio-taped, verbally translated to English and tape-recorded to English prior to transcription. Transcriptions were checked line by line for accuracy before analysis. One translator competent in Hindi or Punjabi was asked to translate and transcribe two interviews to ensure accuracy of translation for credibility. Constant reflexivity and reflection reduced the imposition of preconceived assumptions. Saturation of data was achieved when similar themes became apparent.

Participant observation data and field notes were analyzed concurrently with the interview data and field notes in order to facilitate the understanding of health professionals' work and to provide information about context of the clinical settings. As preliminary data analysis progressed, further participant observations were conducted to clarify, confirm and validate contextual data. Initial coding of the data was done line by line to identify important ideas/experiences. The data inclusive of field notes and documents were analyzed and catalogued by codes into NVivo (7), a software computer program for comparing and contrasting categories. Both specific categories and more abstract themes were constantly refined as new data were generated and more in-depth analysis completed. Data pertaining to similar ideas/experiences were grouped into related categories and synthesized in order to identify important themes that described key aspects of South Asian immigrant women's experiences of respect.

\section{Findings}

Eleven women of Indian ethnicity originally from Fiji Islands, India, Pakistan, and Sri Lanka had been residents of Canada for three to 31 years. Languages varied from English, to Hindi, Urdu, Punjabi, or Tamil. Women's religion varied from Christianity, Hinduism and Muslim to Sikhism. Women ranged in age from 40 to 80 , with the majority being over age 60 . Educational backgrounds ranged from no schooling to university degrees. Most women had retired or had never worked. With the exception of four women who were widowed, all of the women were married. Types of cancer ranged from brain to breast, colon, and tongue.

\section{Meaning of respect}

Women's stories of being respected were anchored in the context of their previous health care experiences, understanding of the cancer journey, and their expectations of respect in light of their heritage and frame of reference for professional relationships. Most women said that personal manifestations of beliefs, values, underlying assumptions, and expectations of respect were related to cultural and societal structures. Women understood that language and words used to describe respect and its attributes might be different because of culture and ethnicity. However, the behaviours or ways of bestowing respect were viewed as similar among women of all ethnicities. Participants consistently responded with the same South Asian term for respect (ijjat) and related its meaning to their experiences of being respected within the health professional-client relationships. Health professionals' genuine attempts to understand their ethnicity and their ability to acknowledge and value women as human beings first and the cancer diagnosis second allowed them to feel at home in a strange environment. This was seen as a basic principle and a very important attribute of respect (ijjat); of being in relationships; an inherent aspect of humanity, and being in social relationships:

Respect means regarding others as persons, as individuals to be acknowledged with kindness and politeness. If you accept others regardless of what or who they are, it is respect. It does not matter what race a person is. It is our way of speaking that is different, e.g., we say "ijjat" (respect in Punjabi) and Canadian women say 'respect'. (3)

Respect is when any human being has come over to your home to visit; you should talk to them with politeness and kindness. You talk with love, and acknowledge them, regardless of who comes to your home. (1)

There was congruence between how South Asian immigrant women treated guests in their own homes and how they envisioned being treated by the health professionals in the clinics. The need to be respected for 'my social identity', as a South Asian immigrant woman with cancer, was woven throughout women's stories and was influenced by their personal life experiences, culture, gender and importance in the family unit where their age demanded respect. Cancer conditions invoked feelings of fear and loss, especially in light of some women's recent migration and transition experiences, along with the fact that their extended family members might be overseas.

I am here by myself since my husband passed away. I stay with my relatives. I might move back to be with my children. (3)

\section{Health professionals' way of being}

Most women shared that being seen as an individual, regardless of age, gender, ethnicity or class empowered them as they dealt with the cancer conditions. By treating women as equals and acknowl- 
edging their shared humanity, health professionals allowed women to have their own perspective, have their own social identity and to contribute holistically to the health professional-client relationship. For women, the ability to express the self was important, regardless of their inability to speak in English when discussing a life-threatening illness that affected their femininity and gender self-perception. Being heard meant they had a voice:

There isn't a difference between the Canadian women or the immigrant women... only the language; we don't have the ability to express ourselves as they can, is a challenge. (5)

My mother tongue, in my language it is easier for me to express myself about my feelings and to earn people's respect. 'I could show them who I am in my own language'. (4)

Being accepted and treated equally was identified as an attribute, whereas greeting was seen as a component of respect, allowing women to feel like they were partners in their cancer journey with the health professional. Women described that health professionals' genuineness when greeting women went beyond mere courtesy; it welcomed them into the health professional-client relationship. Greeting the other was identified as a basic principle of respect and of being in relationships; an inherent aspect of humanity and expectation of being in social relationships. Genuineness when greeting, went beyond mere courtesy, manners or politeness; it acknowledged the woman in the relationship:

It depends on the behaviour and the way that a person talks to you, and then how the person provides care for you. First you have to respect that person by saying "hello" to them and behave in a respectful polite manner, and then you provide care. (4)

\section{Health professionals' way of attending}

Participants shared that concern for privacy and modesty is probably inherent in being a woman. However, the extent of this shyness in relation to the female body might be unique for each woman depending on her past experiences and cultural upbringing, regardless of their age. Most women felt that health professionals preserved the humanity within the health professional-client relationship by respecting their femininity and vulnerability although this was a clinical setting. Most women shared that shyness and modesty was embedded in their South Asian culture due to a lack of exposure to Western style of dress:

My need for privacy may be different from other women's need for it. I want the door and the blinds closed; that is how I am as a South Asian woman. Giving me privacy is respect and regard for my womanhood. That would be the same for women of any ethnicity. (3)

Women felt inherently worthy of respect and attention when health professionals showed genuine concern by taking an open approach that did not always allow for assumptions of cultural taboos of dos and don'ts to interfere with acknowledging the other, especially when health professionals took the time and prayed with women. For most of the women, clinical visits had a special meaning because they experienced human connectedness when health professionals took the time to provide attention and care at a time in their life when they needed it most. One woman who had radiation to her skull described her experiences of feeling respected when the health professional treated her as she would treat a guest in her home. The health professional putting on her toque after the radiation treatment dignified her and made her feel whole again. This story indicated that respect did not need to be created by extravagant details; sometimes, the small things made a difference in the amount of respect, or lack of it:

They helped me on to the stretcher... they put screws on the side of my head, you know for the machine. I have to go underneath the machine, I take off my toque. They respected me... and then helped me put on my jacket and toque. These were behaviours of respect. (10)

\section{Health professionals' way of talking}

Most women felt that because health care experiences located in the institutionalized clinical setting were generally very invasive and affected the whole person who was suffering, greeting them "by say- ing or nodding 'hello'” before provision of care humanized the relationship. However, some women felt brushed off when some physicians were indifferent in their approach and would ask; "What is the problem?" without taking the time to say hello, a universal greeting.

Greetings involved verbal or nonverbal behaviours, such as a nod, a smile, an eye contact, or saying good-bye at the end, giving them a place within the relationship. Subtleness of respect that was determined by the health professionals' appearance, attitude, behaviour or actions at the beginning of the relationship, set the tone for a respectful relationship. Most women felt these behaviours to be a small part of the larger picture that encouraged them to come back for the daily radiation treatments, which may last as long as 35 days:

They always say hello, how are you? Even after the treatment, they help me dress and always say goodbye, see you tomorrow. I felt happy and peaceful in my heart and I wasn't scared of going back for the treatment. (6)

I think the way they are brought up, their whole attitude, beliefs and values toward other people, and the way they think of the other person shows respect. It shows in their actions. (2)

Most women described that health professionals' type of communication style and information delivery respected them and helped put them at ease during these vulnerable times. Women felt that all cancer patients are overwhelmed and have to deal with a host of things, such as their mortality, the possibility of leaving their loved ones behind and having to make decisions about their employment and quality of life in relation to the cancer-related treatments and its side effects. This younger woman of 40 with a child felt respected when health professionals made a conscious effort to engage with her when providing explanations about her cancer condition and valuable information about her body:

When you find out about cancer, your brain is all occupied, thinking of all the stuff, you know. However, I felt so safe... they explained the cancer information. I was not scared; I felt free. (7)

In contrast, other women felt that some health professionals', mostly physicians', way of talking and engaging with them during the interactions either closed the door or opened it to further interactions, especially when they used complex medical terminology. It was not only the quantity and quality of information that was provided, but also the quality of the delivery, the communication style, which enhanced women's experiences of respect:

He was talking in his medical terms... the right word. Sometimes you think he doesn't want to pay attention, but it is just his terminology he uses; it is not his personality. (8)

Because some women did not have an opportunity to speak English regularly, they felt frustrated when they had difficulty expressing themselves in relation to their health care experiences. This feeling of frustration was not because of women's inability to speak the English language, but because they could not find the right words, phrase or terms to describe what they were feeling. This marginalized them during their interactions, because they were unable to speak from their heart about their suffering and pain related to the cancer condition. Some women shared that at times they did not want to use English because the suffering, fatigue and pain took up so much of their precious energy, which they needed to perform the activities of daily living. This woman shared that it was an effort to remember how to use English during times of suffering:

Usually I understand English, but when there is a lot of pain involved then I don't want to speak in English. I don't want to speak/think. If they speak slowly then I understand. (1)

Most women were thankful and felt respected during interactions with interpreters, as the interpreters provided information and explanations about treatments. However, some health professionals were unaware that South Asian immigrant women speak different languages based on the subgroups to which they belong. These assump- 
tions on the health professionals' part made it difficult for women to comprehend the information. Some women who spoke Hindi, for example, were provided with Punjabi-speaking interpreters:

In the beginning...was an Indian woman who explained in Hindi. You know some Punjabi women speak Hindi, but most only speak Punjabi and it is hard to understand them. (9)

Most of the time there are interpreters. Sometimes there isn't an interpreter, but my sister-in-law speaks English [the sister-in-law sometimes accompanies her to act as an interpreter, but mostly she has to rely on the interpreter provided by the hospital]. I may not be able to speak English, but I understand the doctor. I cannot explain myself, so I have to speak to the interpreter in Hindi. (11)

These women felt that some health professionals may have some preconceived assumptions leading to stereotyping women, which reduced respect and created barriers in communicating pertinent information regarding cancer treatments. Women suggested that health professionals should offer a more individualized approach and ask women about their ability to speak English regardless of the women's age or ethnicity. Participants suggested that health professionals should initially ask family members or women what language they preferred or spoke and then provide an interpreter who speaks the same language as the patient. Because most South Asian patients being cared for at these clinics spoke Punjabi, Hindi or Urdu as their first language and English as the second, this strategy would help patients' enhance their understanding of cancer. Furthermore, women also felt this approach to the health professional-client relationship would reduce assumptions and stereotyping and increase respect:

The interpreter was an Indian Punjabi woman, but she spoke Hindi to me. Some Punjabi women speak Hindi very well, others do not. Also, most Punjabi women speak very deep in their own language, so it is hard to understand them. In the chemotherapy clinic there are some Punjabi nurses, but one of them does not understand Hindi. (2)

Although most women preferred discussing issues related to the female reproductive systems with female health professionals, they were resigned to being cared for by male or female health professionals. However, most women experienced that having female interpreters who spoke their own language provided them with a sense of comfort because they could speak to the 'male health professional' through the 'female interpreter' who spoke their language.

Consequences of respect bestowed by health professionals' during clinical visits were that women felt safe and comfortable, regardless of the ambiguity of the cancer condition. Most women felt "at home" in the clinical environment regardless of their ability to speak English because of the way health professionals "talked to them." Respecting a fellow human being through recognition and greeting the other regardless of his or her position in society, religion, gender, age, culture or health status is an inherent quality that values the other's social identity.

\section{Discussion}

The central theme "who I am as an individual" was consistent in women's stories of being recognized as individuals with a social identity, a standpoint. The complexity of providing care for culturally diverse clients can be addressed by creating safe environments in which frank but respectful discussions within health professional-client relationships can take place. Browne's (1995) study with a First Nations population confirms findings that a link between health care providers' attitudes and behaviour toward them as First Nations women and the incidence of respectful clinical interactions created feelings of equality and worthiness. Clegg (2003) shared how the concept of respect for the individual was influenced in global terms by the political, social, economic and religious interpretations.

Johnson et al. (2004) in their study about "othering" in health care services with South Asian immigrant women, noted the need for values such as equality and respect for diversity within the health care setting. However, these notions might sometimes be used in ways that obscure the very real barriers and disadvantages that most immigrants face in accessing health care and adjusting to life in Canada. Vydelingum (2006) reported that by seeing only the differences within the "other" cultures, health professionals' tended to practise a reductionist approach that allowed the nurses to view immigrant women "as a series of 'dos' and 'don'ts"” (p. 29) rather than as people with their own identity.

Providing culturally appropriate care prompts us to question our own assumptions about the cultural groups we are caring for, to locate women's health concerns within a structural context, and to connect the dynamics of individual encounters to broader social, political and economic issues (Browne, 2003; Harris \& Templeton, 2001; Spitzer et al., 2003). Hilton et al. (2001), in their study with South Asian immigrant women in Canada, confirmed that respect for women's need to practise their traditional home remedies provided a way to "maintain and preserve their cultural heritage and identity as a South Asian woman" (p. 558). Bottorff, Johnson, et al. (1998), in a study of South Asian women's beliefs related to breast cancer, highlighted the need to respect and recognize the beliefs concerning a woman's calling and her modesty related to how she presents herself in society, and how she is to act in society and within the community.

Findings in the current study reveal that health professionals' ability to communicate respect through genuine and spontaneous greetings by interacting with women on an individualized basis at the beginning of the health professional-client relationship helped to give it a "person-centred" approach, whereby identity was more important than ethnicity. Similar findings emphasized openness in communication and genuine concern through acknowledgement at the beginning of an encounter, as part of building a caring professional health care relationship that left patients feeling more like whole persons whose psychosocial, physical and emotional needs had been met (Liu, Mok, \& Wong, 2005; Thorne et. al., 2005).

The current study revealed a lack of insight on the part of the health professionals and the clinic concerning the range of South Asian languages spoken. Furthermore, policies and guidelines on the procedures of interpretive services provided by the clinic may need to be examined closely in order to increase an awareness of various sub-groups within the South Asian communities who may speak different languages. Anderson (1998) has reported the need to challenge existing health care policies related to interpretive services and language-specific materials for diverse populations. Fitch et al. (1998) indicated that oncology nurses faced diverse challenges in the clinical setting while providing care and administering complex cancer-related treatments, and these challenges were heightened when the nurses were working with immigrants who might be non-English speaking. Findings from other studies have recognized that in institutions that serve a large immigrant population, interpreters could function as cultural cancer mediators and advocates, thereby decreasing emotional stress and exhaustion and improving health care relationships, especially if they were part of the institution, making them familiar with existing health policies and procedures (Singh, 2002; Vydelingum, 2006).

Raising awareness that the patient is 'another' human being within the relationship can be achieved through critical self-awareness of the health professional's own culture and recognition of his or her biases, which can be learned and fostered to counter stereotyping of the 'other' population. In addition, tailoring communication style to fit with the patient, the type of cancer, and its related treatments will be seen as creating respectful and satisfying relationships for both the health professional and the client (Bottorff, et al., 2007).

\section{Conclusion}

Because this study was limited to South Asian immigrant women, the findings may not apply to women of other ethnicities or to men. Diversity among participants' level of income and education with 
variations in women's' age may provide different perspectives of respect. To some extent, the relatively small number of interviews conducted for this study might be a limiting factor. However, the diversity of the subgroups helped to increase the representation of the population under study. This study also gained strength from the diversity of participants' types of cancer experiences and participant observations of all the health care disciplines' provision of care for clients at the clinical setting. It may be useful in future studies to examine women's perceptions of respect within relationships between nurses and other health professionals at the clinic. Findings of this research suggest the potential merit for further research with vulnerable populations and health professionals' communication styles when providing cancer care, especially when communication underpins partnership in cancer care.

\section{Acknowledgements}

The authors thank all the participants and the oncology nurses who contributed to this research.

\section{References}

Acton, G.J., Irvin, B.L., Jensen, J A., Hopkins, B.A., \& Miller, E.W. (1997). Explicating middle-range theory through methodological diversity. Advances in Nursing Science, 19(3), 78-85.

Anderson, J.M. (1998). Speaking of illness: Issues of first generation Canadian women-Implications for patient education and counselling. Patient Education and Counselling, 33, 97-207.

Barbosa, I.A., \& Silva, M.J. (2007). Humanization in nursing care: Acting with respect in a school hospital. Revista Brasileira de Enfermagem, 60, 546-51.

Beauchamp, T.L., \& Childress, J.F. (1994). Principles of biomedical ethics (4th ed.). New York: Oxford University Press.

Benner, P. (2002). Creating compassionate institutions that foster agency and respect. American Journal of Critical Care, 11, 164-166.

Bottorff, J.L., Grewal, S., Balneaves, L.G., Naidu, P., Johnson, J.L., \& Sawhney, R. (2007). Punjabi women's stories of breast cancer symptoms. Cancer Nursing, 30(4), E36-E45.

Bottorff, J.L., Johnson, J.L., Bhagat, R., Grewal, S., Balneaves, L.G., Clarke, H., et al. (1998). Beliefs related to breast health practices: The perception of South Asian women living in Canada. Social Sciences \& Medicine, 47, 2075-2098.

Browne, A.J. (1995). The meaning of respect: A First Nation's perspective. Canadian Journal of Nursing Research, 27(4), 95-109.

Browne, A.J. (1997). A concept analysis of respect: Applying the hybrid model in cross-cultural settings. Western Journal of Nursing Research, 19, 762-780.

Browne, A.J. (2003). First Nations women and health care services: the socio-political context of encounters with nurses. Unpublished doctoral dissertation, University of British Columbia, Vancouver, BC, Canada.

Canadian Cancer Society/National Cancer Institute of Canada. (2008). Canadian Cancer Statistics, Toronto, Canada, 2008. http://www.cancer.ca/canada-wide/about\%20cancer/cancer\%20 statistics/canadian\%20cancer\%20statistics.aspx?sc_lang=en

Clegg, A. (2003). Older South Asian patient and carer perceptions of culturally sensitive care in a community hospital setting. Journal of Clinical Nursing, 12, 283-290.

Erlen, J.A. (1998). Culture, ethics, and respect: The bottom line is understanding. Orthopaedic Nursing, 17(6), 79-82.

Fitch, M.I., Greenberg, M., Cava, M., Spaner, D., \& Taylor, K. (1998). Exploring the barriers to cervical screening in an urban Canadian setting. Cancer Nursing, 21, 441-449.

Gallagher, A. (2007). The respectful nurse. Nursing Ethics, 14, 360-371.

Gupta A., Kumar A., \& Stewart, D.E. (2002). Cervical cancer screening among South Asian women in Canada: The role of education and acculturation. Health Care for Women International, 23, 123-134.

Harris, S.R., \& Templeton E. (2001). Who's listening? Experiences of women with breast cancer in communicating with physicians. Breast Journal, 7, 444-449.

Hilton, B.A., Grewal, S., Popatia, N., Bottorrf, J.L., Johnson J.L., Clarke, H., et al. (2001). The desi ways: Traditional health practices of South Asian women in Canada. Health Care for Women International, 22, 553-567.
Hislop, T.G., Bajdik, C.D., Saroa. S.R., Yeole B.B., \& Barroetavena M.C. (2007). Cancer incidence in India from three areas: Delhi and Mumbai, India, and British Columbia, Canada. Journal of Immigrant and Minority Health, 9, 221-227.

Johnson, J.L., Bottorff, J.L., Browne, A.J., Grewal, S., Hilton, B.A., \& Clarke, H. (2004). Othering and being othered in the context of health care services. Health Communication, 16, 253-271.

Kelly, B. (1990). Respect and caring: Ethics and the essence of nursing. In M.M. Leininger (Ed.), Ethical and moral dimensions of care (pp. 213-221). Detroit, MI: Wayne State University Press.

Kirkham, S. (2003). The politics of belonging and intercultural health care. Western Journal of Nursing Research, 25, 762-780.

Klutgen, J. (1995). Autonomy and intervention. New York: Oxford University Press.

Liu, J.E., Mok, E., \& Wong, T. (2005). Perceptions of supportive communication in Chinese patients with cancer: Experiences and expectations. Journal of Advanced Nursing, 52, 262-270.

Milton, C.L., (2008). Boundaries: Ethical implications for what it means to be therapeutic in the nurse-person relationship. Nursing Science Quarterly, 21, 18-21.

Morris, N.M. (1997). Respect: Its meaning and measurement as an element of patient care. Journal of Public Health Policy, 18, 133-151.

Morse, J.M., \& Field, P.A. (1995). Qualitative research methods for health professionals (2nd ed.). Thousand Oaks, CA: Sage.

Peters, R.S. (1961). Respect for persons and fraternity. In R.S. Peters (Ed.), Ethics and education (pp. 133-137). Palo Alto, CA: Scott \& Foresman.

Rokeach, M. (1979). Understanding human values. New York: Free Press.

Roper, J.M., \& Shapiro, J. (2000). Ethnography in nursing research. Thousand Oaks, CA: Sage.

Singh, S.W. (2002). An exploration of South Asian women's experiences following abnormal pap smear results. Unpublished master's thesis, University of British Columbia, Vancouver, BC, Canada.

Smith, D.E. (1987).The everyday world as problematic: A feminist sociology. Boston: North Eastern University Press.

Spitzer, D., Neufeld, A., Harrison, M., Hughes, K., \& Stewart, M. (2003). Care giving in the transnational context: "My wings have been cut: where can I fly?" Gender and Society, 2(10), 1-20.

Statistics Canada. (n.d.). 2007 census of population-immigrant population by place of birth and period of immigration. Retrieved from http://www.statscan.ca/english

Tarlier, D.S. (2004). Beyond caring: The moral and ethical bases of responsive nurse-patient relationships. Nursing Philosophy, 5, 230-241.

Thorne, S.E, Bultz, B.D., \& Baile, W.F. (2005). Is there a cost to poor communication in cancer care?: A critical review of the literature. Psycho-Oncology, 14, 875-884.

Vydelingum, V. (2006). Nurses' experiences of caring for South Asian minority ethnic patients in a general hospital in England. Nursing Inquiry, 13, 23-32.

Widäng, F., Fridlund, B., \& Martensson, J. (2007). Women patients' conceptions of integrity within health care: A phenomenographic study. Journal of Advanced Nursing, 61, 540-548. 